\title{
Long-term outcomes of different endoscopic sinus surgery in recurrent chronic rhinosinusitis with nasal polyps and asthma*
}

\author{
Luo Zhang 1,2,3,\#, Yuan Zhang 1,2,3,\#, Yunbo Gao', Kuiji Wang' ', Hongfei Lou', \\ Yifan Meng', Chengshuo Wang' \\ ' Department of Otolaryngology Head and Neck Surgery, Beijing TongRen Hospital, Capital Medical University, Beijing, China \\ 2 Beijing Key Laboratory of Nasal Diseases, Beijing Institute of Otolaryngology, Beijing, China \\ ${ }^{3}$ Department of Allergy, Beijing TongRen Hospital, Capital Medical University, Beijing, China
}

Rhinology 58: 2, $126-135,2020$

https://doi.org/10.4193/Rhin19.184

*Received for publication:

May 2, 2019

Accepted: November 28, 2019

\# contributed equally to this work

\begin{abstract}
Background: Patients with chronic rhinosinusitis with nasal polyps (CRSwNP) and asthma have poorer outcomes after functional endoscopic sinus surgery (FESS) and higher recurrence rate. The aim of present study was to investigate the long-term clinical outcomes of extended surgical strategies for patients with recurrent CRSwNP and asthma.
\end{abstract}

Methods: Eighty-one patients with CRSwNP and asthma were enrolled in this 5-year prospective study. They were randomly assigned to undergo FESS, radical endoscopic sinus surgery (RESS), or RESS+Draf 3 surgery. Disease severity and clinical outcomes were evaluated using symptoms scoring, endoscopic scoring system, computed tomography staging system, sinus-specific quality of life scores, tissue and peripheral blood eosinophil percentage, and pulmonary function tests. Baseline, 1-year, 3-year, and 5-year follow-up data were compared among the groups.

Results: RESS and RESS+Draf 3 strategies yielded better short-term (1 year) outcomes than did FESS. FESS had a higher short-term recurrence rate, although recurrence rates were similarly high (95.6-96.1\%) in all the groups at 5 years postoperatively. RESS and RESS+Draf 3 yielded a lower long-term revision surgery rate and a longer time to recurrence post-surgery than FESS, which was negatively correlated with tissue and peripheral blood eosinophil percentage.

Conclusions: CRSwNP with asthma is a systemic disease that inevitably recurs. Radical surgery prolongs recurrence time and improves olfaction, rhinorrhea, and quality of life in the short-term. Combining Draf 3 with RESS did not yield better clinical outcomes than RESS alone; thus, although RESS alone appears to be the best option, these findings need to be confirmed in further studies involving more patients, longer follow-up duration and stricter standardized medication use especially the adequate steroid irrigations.

Key words: chronic rhinosinusitis, Draf 3, eosinophils, nasal polyps, surgery

\section{Introduction}

Chronic rhinosinusitis (CRS) remains a common, challenging clinical entity; it has variable phenotypes with different underlying mechanisms, which lead to persistence or recurrence of nasal polyps (NPs). CRS is divided into CRS without NP (CRSsNP) and CRS with NP (CRSwNP) $)^{(1)}$. The predominant inflammatory patterns are thought to influence the clinical manifestations and responses to current medical and surgical interventions ${ }^{(2 .}$ 3). CRSwNP with asthma is considered to be the most refractory CRS type, which easily relapses, is very difficult to treat ${ }^{(4,5)}$.Longterm management with steroids is likely to be the mainstay strategy for these patients ${ }^{(6,7)}$, although they still show a strong 
tendency for recurrence (up to 55.3\%) and subsequent revision surgery ${ }^{(2,3,8)}$. To date, there is no consensus on the optimal surgical strategy for such patients.

Since the concept of "functional endoscopic sinus surgery (FESS)" was introduced ${ }^{(9-12)}$, surgical treatments for CRS have advanced, and surgery is considered a standard treatment for CRSwNP. Yet, this approach might be inadequate and is not appropriate for all cases, specifically those with refractory NPs. Bachert et al. thus proposed to differentiate surgical approaches on the basis of the inflammatory endotypes ${ }^{(13)}$. Jankowski et al. reported that the recurrence rate with radical endoscopic sinus surgery (RESS) was decreased significantly compared to FESS ${ }^{(14)}$. Chen et al. also revealed that extensive endoscopic sinus surgery (EESS) for patients with concomitant CRSwNP and asthma may improve subjective olfaction and endoscopic appearance ${ }^{(15)}$. Additionally, a recent study reported that complete sphenoethmoidectomy, maxillary antrostomy, and the endoscopic modified Lothrop procedure (EMLP) (Draf 3 ) is successful in most patients with aspirin-exacerbated respiratory disease (AERD) and CRSwNP, with low complication rates. This approach facilitates successful ongoing medical management of the condition in patients with $A E R D^{(16)}$.

To clarify the significance of radical surgery for CRSWNP with asthma, we compared the long-term outcomes of FESS, RESS, and RESS+Draf 3 in patients with recurrent CRSwNP with asthma.

\section{Materials and methods}

\section{Patients and study design}

The study was approved by the medical ethics committee of Beijing Institute of Otolaryngology and Beijing TongRen Hospital, and written informed consent was obtained from each patient before participation. The clinical trial registration details were available from https://www.clinicaltrials.gov/ (NCT03878355). This prospective cohort study used data from patients with bilateral CRSwNP scheduled to undergo endoscopic sinus surgery in the Rhinology Department of Beijing TongRen Hospital between January 1, 2010 and October 31, 2013. At the screening visit, 112 participants with bilateral CRSwNP were preselected as candidates; of whom 31 patients were excluded because they did not meet the following inclusion criteria: having had at least 1 previous sinus surgery (FESS, full maxillary antrostomy, ethmoidectomy, sphenoidotomy and frontal sinusotomy, but with the middle turbinate [MT] preservation), "good gasification" of the frontal sinus (i.e a developed frontal ostium with anteriorposterior diameter $\geq 10 \mathrm{~mm}$,) and with no complicated frontal sinus mucocele or tumor to warrant a successful Draf $3^{(17,18)}$, with concomitant asthma, with no unilateral disease, allergic fungal rhinosinusitis, antrochoanal polyps, or cysts, and treated with no antibiotics or corticosteroids within a 4-week period before enrollment. The extensiveness of previous FESS surgery was confirmed on the basis of hospital records of the surgery and performance of a CT scan on enrolment to the study. The diagnosis of CRSwNP was based on the standard criteria of the European Position Paper on Rhinosinusitis and Nasal Polyps guidelines (EPOS) $)^{(19)}$. All recruited subjects met the criteria for refractory $\mathrm{CRS}^{(19)}$. Asthma diagnosis was confirmed by a chest physician according to Global Initiative for Asthma (GINA) guidelines ${ }^{(20)}$. The 81 patients meeting the inclusion criteria between January 1, 2010 and October 31, 2013 were randomized to undergo FESS, RESS, or RESS+Draf 3 surgery. Randomization was performed in sets of three consecutive patients (i.e. in a ratio of 1:1:1) according to a computer-generated randomization code. Following surgery, all subjects completed a minimum 5-year follow-up until December 31 2018; however, the first patients who underwent surgery at the beginning of the study in January 2010 were followed-up for a total of nine years up to December 31,2018 and the last patients undergoing surgery in October 2013 being followed-up for five years up to December 312018. All patients were followed-up at 3 monthly intervals over the entire postsurgery period for evaluation of polyp recurrence and revision surgery status. Furthermore, all patients were also evaluated at 1, 3 and 5-year postoperative follow-ups were performed for polyp recurrence, revision surgery status, symptom scores, endoscopic scores, SNOT-22, CRSwNP, as well as clinical control of asthma.

\section{Surgical procedures}

All surgical procedures were performed under general anesthesia by the same senior rhinology specialist (C.W.), thereby reducing the risk of inconsistency or bias for a particular procedure. FESS was performed using the Messerklinger technique ${ }^{(9)}$, and involved full maxillary antrostomy, ethmoidectomy, sphenoidotomy and frontal sinusotomy, but with MT preservation. Similarly, RESS was undertaken using "full-house FESS (FHF)" approach, and involved complete removal of all NPs along with full maxillary antrostomy, total ethmoidectomy, wide sphenoidoto$\mathrm{my}$, and a Draf $2 \mathrm{~A}$ frontal sinusotomy ${ }^{(21-23)}$. Moreover, the bilateral inferior two-thirds of MTs were resected ${ }^{(15)}$. The Draf 3 frontal drillout procedure was performed with resection of the superior nasal septum, central frontal sinus floor, and frontal beak region, resulting in a wide, patent, oval-shaped, common frontal sinus neo-ostium ${ }^{(24)}$. The unaffected mucosa-sparing approach was adopted for all 3 surgical procedures.

\section{Postoperative medical treatment}

A 10-day course of oral antibiotics was completed according to the findings of a microbial culture from a preoperative secretion swab taken from the patient on admittance to the hospital one week before the operation. All patients had a 3-week-tapering course of oral methylprednisolone (initial dose: 24 mg, Medrol, Pfizer, Rome, Italy) postsurgically. Budesonide nasal spray 
Table 1. Demographic data and preoperative disease burden of patients undergoing FESS, RESS, and RESS+Draf 3.

\begin{tabular}{|c|c|c|c|c|}
\hline Parameter & RESS+Draf $3(n=27)$ & EESS $(n=27)$ & FESS $(n=27)$ & $\mathbf{P}$ \\
\hline Age (years), mean (SD) & $47.30 \pm 11.03$ & $41.37 \pm 12.76$ & $44.56 \pm 11.23$ & 0.18 \\
\hline Sex (male/female), $n$ & $19 / 8$ & $14 / 13$ & $15 / 12$ & 0.34 \\
\hline Allergy, n (\%) & $10(37.0 \%)$ & $9(33.3 \%)$ & $7(25.9 \%)$ & 0.67 \\
\hline Aspirin-sensitive, n (\%) & $8(29.6 \%)$ & $7(25.9 \%)$ & $9(33.3 \%)$ & 0.84 \\
\hline Smoker, n (\%) & $4(14.8 \%)$ & $4(14.8 \%)$ & $4(14.8 \%)$ & 1.00 \\
\hline Previous FESS surgery, $\mathrm{n}(\%)$ & $1.41 \pm 0.64$ & $1.37 \pm 0.56$ & $1.37 \pm 0.63$ & 0.97 \\
\hline \multicolumn{5}{|l|}{ Symtom scores, median (IQR) } \\
\hline Congestion & $8.0(7.7-8.5)$ & $8.0(7.5-8.4)$ & $8.0(7.8-8.4)$ & 0.4535 \\
\hline Rhinorrhea & $5.0(4.0-6.0)$ & $5.0(4.0-5.0)$ & $4.0(4.0-5.0)$ & 0.30 \\
\hline Loss of smell & $9.0(9.0-10.0)$ & $10.0(9.0-10.0)$ & $10.0(9.0-10.0)$ & 0.38 \\
\hline Head/facial pain & $3.0(2.0-4.0)$ & $3.0(3.0-3.0)$ & $3.0(3.0-3.0)$ & 0.89 \\
\hline SNOT-22 & $64.0(54.0-75.0)$ & $65.0(57.0-68.0)$ & $64.0(61.0-67.0)$ & 0.99 \\
\hline Polyp score & $5.0(4.0-5.0)$ & $5.0(4.0-5.0)$ & $5.0(4.0-5.0)$ & 0.47 \\
\hline Lund-Mackay score & $22.0(18.0-24.0)$ & $20.0(17.0-24.0)$ & $20.0(18.0-23.0)$ & 0.70 \\
\hline Blood eos $\%$ & $8.40 \pm 3.54$ & $8.21 \pm 4.90$ & $9.46 \pm 5.85$ & 0.60 \\
\hline Tissue eos\% & $69.98 \pm 7.27$ & $69.71 \pm 9.99$ & $68.39 \pm 10.40$ & 0.56 \\
\hline
\end{tabular}

FESS: functional endoscopic sinus surgery; RESS: radical endoscopic sinus surgery; IQR: inter-quartile range.

(Rhinocort Aqua, AstraZeneca, Södertälje, Sweden) $128 \mu \mathrm{g}$ twice a day and normal saline solution nasal lavage were commenced after surgery; with the Budesonide nasal spray being administered continuously for at least 6 months until patients achieved good clinical control of CRS, as defined by the EPOS 2012 guidelines $^{(1)}$. When polyps or mucosal edema were noted postoperatively, intranasal steroid treatment was commenced. In cases where more than three months' intranasal steroid usage did not control mucosal edema and inflammation, oral methylprednisolone was used. Clarithromycin $250 \mathrm{mg}$ was used daily for 3 months for persistent infections and/or concurrent neutrophil inflammation in the nasal cavity. Antihistamine and/ or leukotriene antagonists were used when the patient was diagnosed with allergic rhinitis (AR) and the related AR symptoms could not be controlled by steroid nasal spray alone. Beyond this, the main strategy for therapy was to take medication on demand, based on the actual symptoms and endoscopic scores at the 3 monthly follow-up visits over the entire five years period post-surgery.

\section{Clinical assessment}

To avoid potential bias by the surgeon performing the initial endoscopic surgery, a second rhinology specialist (Y.Z.) independently assessed the clinical outcomes. Subjective symptoms including nasal congestion, rhinorrhea, loss of smell, and headache and/or facial pain were scored on a 0-10 integer points. Sinus-specific quality of life (QoL) was assessed using the 22-item Sinonasal Outcome Test (SNOT-22) ${ }^{(25)}$. The preoperative polyp score was graded for each nasal cavity on a scale of $0-3$ for each side, and the bilateral polyp grade was the sum of the individual units for the left and right nasal cavities (maximum, 6) as previously described ${ }^{(26)}$. Postoperatively, endoscopic results were scored according to the Lund-Kennedy system, with assessment of edema, nasal discharge, scarring, and crusting ${ }^{(27)}$. Computed tomography (Philips Health Care, Best, The Netherlands) of paranasal sinuses was performed in all patients presurgically, and was scored using the Lund-Mackay system ${ }^{(28)}$. Allergy status was confirmed based on Immuno-Cap Phadiatop (Pharmacia, Uppsala, Sweden) (cutoff $\geq 0.35 \mathrm{kU} / \mathrm{mL}$ ). Asthma was evaluated by pulmonary function tests, using the percentage forced expiratory volume in 1 second (FEV1\%) assessed spirometrically (MasterScreen, Jaeger, Germany) and a FEV1\% of $<80 \%$ was graded as abnormal ${ }^{(29)}$. Clinical control of CRS was graded in terms of EPOS 2012 guidelines ${ }^{(1)}$. and asthma was assessed in reference to the GINA guidelines ${ }^{(20)}$. Similarly, aspirin sensitivity was defined according to the EPOS 2012 guidelines ${ }^{(1)}$, based on positive oral, bronchial, or nasal provocation tests, or an obvious history. In cases where more than two courses of oral methylprednisolone (3-week-tapering course with $24 \mathrm{mg}$ initial dose) combined with 3 months Clarithromycin ( $250 \mathrm{mg}$ daily) usage did not control mucosal edema and inflammation (polyp score $\geq 2$ ) and nasal symptoms (at least one symptom score $\geq 7$, i.e. severe symptom), the decision for renewed surgery will be made. 


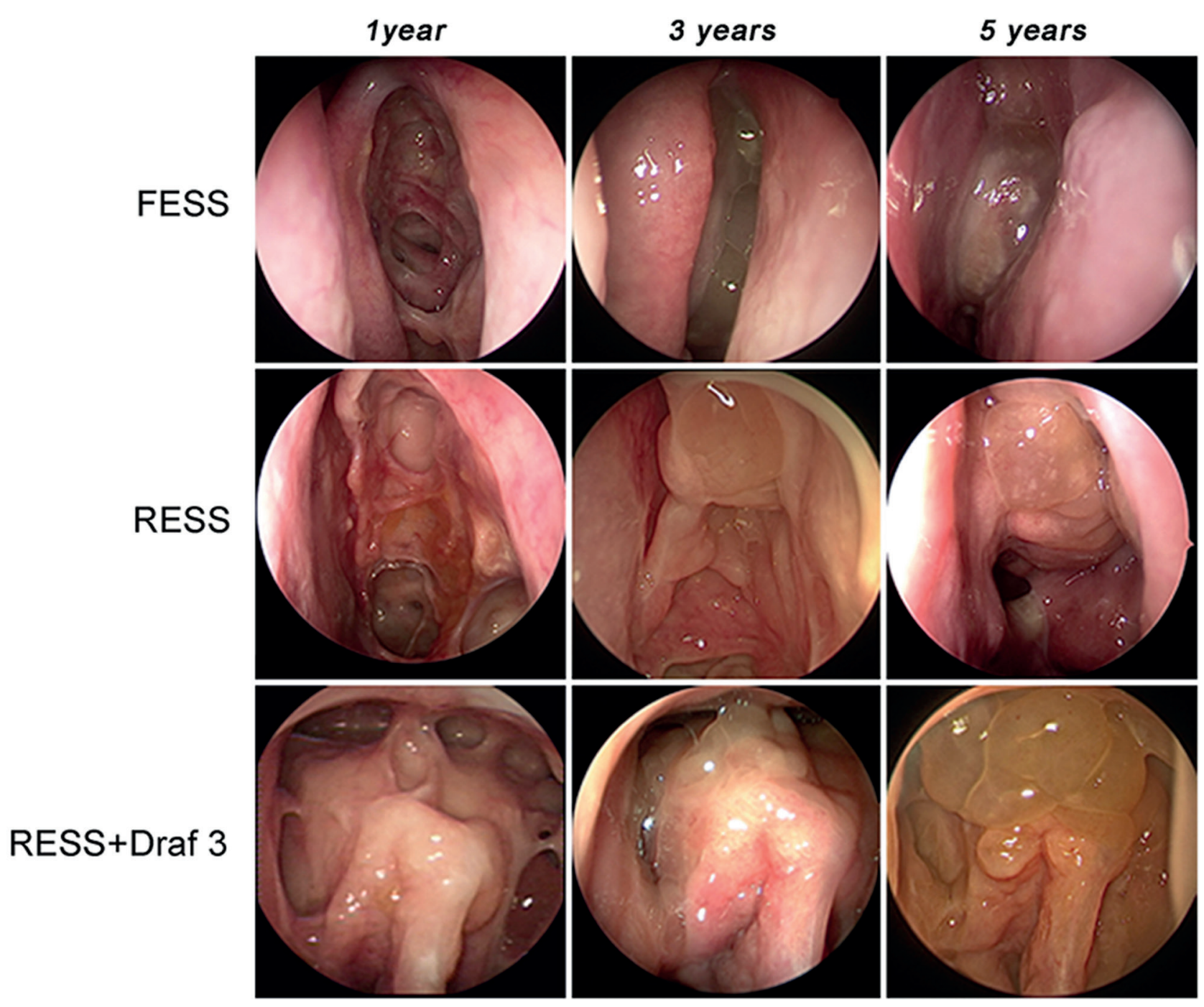

Figure 1. Typical endoscopic views of patients who underwent FESS, RESS, and RESS+Draf 3 obtained at 1 year, 3 years, and 5 years postoperatively. FESS: functional endoscopic sinus surgery; RESS: radical endoscopic sinus surgery.

\section{Statistical analysis}

Statistical analysis was performed in SPSS version 19.0 (IBM Corp, Armonk, NY, USA) and presented using GraphPad Prism software version 7.0 (GraphPad, La Jolla, CA, USA). Discrete variables are reported as frequency and percentage, and continuous variables as median and interquartile range (IQR), unless otherwise specified. Chi-square tests were used for comparison of discrete variables. Kruskal-Wallis tests were used to assess significant intergroup variations. The effect size was calculated by sample size based on nearly $100 \%$ power and false rate at 0.05 . Correlations between recurrence time and percentage of tissue or blood eosinophils were evaluated by Spearman's $r$ test. Kaplan-Meier curves were used to show recurrence-free survival in different groups. Multivariate Cox proportional hazards models, with the duration of recurrence as the underlying time metric, were prepared to estimate the risk of recurrence of CRS associated with potential predictors. All statistical tests were 2-sided, and a level of 0.05 was considered statistically significant.

\section{Results}

Demographics and baseline disease burden

Eighty-one patients with eCRSwNP were enrolled in this prospective cohort study, and 27 individuals each were assigned to the FESS, RESS, and RESS+Draf 3 groups, respectively. One patient in the FESS group, 4 patients in the RESS group, and 2 patients in the RESS+Draf 3 group dropped out because of nonadherence. Even if a patient only completed the 1-year and/ or 3-year follow-up, the data were used in analysis.

There were no significant differences in baseline characteristics, including age, sex, allergy status, aspirin sensitivity, smoking status, number of previous sinus surgeries, symptom scores, SNOT-22, polyp, and Lund-Mackay scores, and percentage of eosinophils in the peripheral blood and polyp tissue (Table 1). Actually, all samples coincided with criterion for eosinophilic 
A
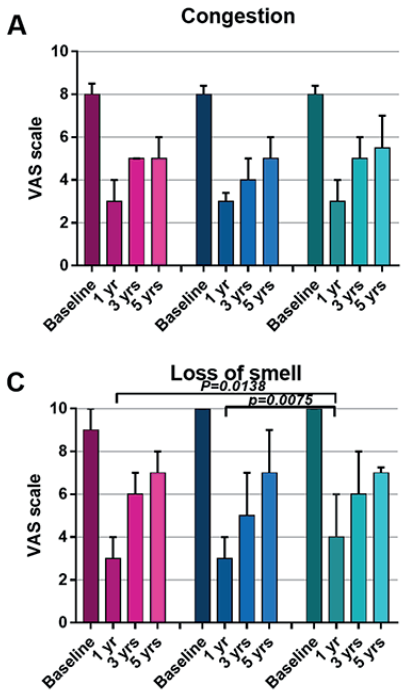

E
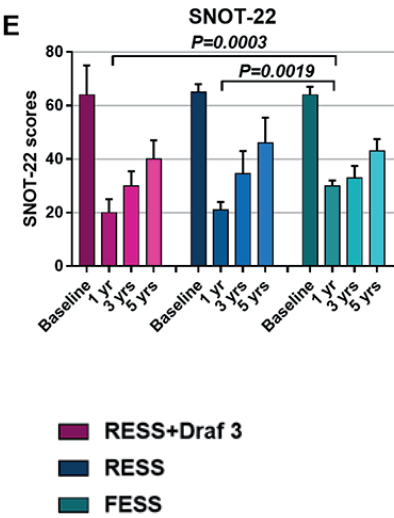

Figure 2. Symptoms, SNOT-22, and endoscopic scores of patients undergoing FESS, RESS, and RESS+Draf 3 at baseline, 1 year, 3 years, and 5 years postoperatively. FESS: functional endoscopic sinus surgery; RESS: radical endoscopic sinus surgery. The bars and error bars represent median and interquartile range, respectively.

CRSwNP (eCRSwNP), i.e. tissue eosinophil count equal to or greater than $54.5 \%$ in polyp tissue ${ }^{(3)}$.

Symptoms, QoL, and endoscopic scores changes in the 3 groups

When comparing symptomatic scores among the 3 groups at baseline and 1 year, 3 years, and 5 years postoperatively, rhinorrhea in the RESS+Draf $3(P<0.001)$ and RESS groups $(P<0.001)$ was relieved more significantly than that in the FESS group at 1 year postoperatively. Patients in both the RESS+Draf $3(P=0.01)$ and RESS groups $(P=0.01)$ had significantly decreased olfactory score compared to the FESS group at 1 year postoperatively. Moreover, power analysis demonstrated that the samples had nearly $100 \%$ power to detect the effect size (f2) 0.55 for rhinorrhea and loss of smell ( $f 2=0.427$ ); at a false rate $(a)=0.05$. There were no differences in nasal congestion and head/facial pain among the 3 groups at this time-point. Moreover, the 4 clinical
Table 2. Five-year recurrence rate and revision surgery rate of patients who underwent FESS, RESS, and RESS+Draf 3.

\begin{tabular}{|c|c|c|c|c|}
\hline Parameter & $\begin{array}{l}\text { RESS+ } \\
\text { Draf } 3\end{array}$ & RESS & FESS & $\mathbf{P}$ \\
\hline \multicolumn{5}{|l|}{$\begin{array}{l}\text { Recurrence rate, } \\
\text { No. }(\%)\end{array}$} \\
\hline 1 year & $\begin{array}{c}16 / 27 \\
(59.3 \%)\end{array}$ & $\begin{array}{c}17 / 27 \\
(63.0 \%)\end{array}$ & $\begin{array}{c}24 / 27 \\
(88.9 \%)\end{array}$ & 0.03 \\
\hline 3 year & $\begin{array}{c}24 / 25 \\
(96.0 \%)\end{array}$ & $\begin{array}{c}22 / 23 \\
(95.6 \%)\end{array}$ & $\begin{array}{c}25 / 26 \\
(96.1 \%)\end{array}$ & $>0.99$ \\
\hline 5 year & $\begin{array}{c}24 / 25 \\
(96.0 \%)\end{array}$ & $\begin{array}{c}22 / 23 \\
(95.6 \%)\end{array}$ & $\begin{array}{c}25 / 26 \\
(96.1 \%)\end{array}$ & $>0.99$ \\
\hline \multicolumn{5}{|l|}{$\begin{array}{l}\text { Revision surgery } \\
\text { rate, No. (\%) }\end{array}$} \\
\hline 1 year & 0 & 0 & 0 & - \\
\hline 3 year & $2 / 25(8.0 \%)$ & $0 / 23$ & $\begin{array}{c}10 / 26 \\
(38.5 \%)\end{array}$ & $<0.001$ \\
\hline 5 year & $\begin{array}{c}4 / 25 \\
(16.0 \%)\end{array}$ & $\begin{array}{c}4 / 23 \\
(17.4 \%)\end{array}$ & $\begin{array}{c}12 / 26 \\
(45.2 \%)\end{array}$ & 0.02 \\
\hline
\end{tabular}

FESS: functional endoscopic sinus surgery; RESS: radical endoscopic sinus surgery.

symptoms did not differ among the groups at 3 years and 5 years postsurgery. SNOT-22 scores showed that RESS+Draf 3 (mean and range: 20: 16 25) or RESS (21: 18 24) patients had more significantly improved QoL than FESS (30: 21 32) patients $(\mathrm{P}<0.001$ and $\mathrm{P}=0.002$, respectively), at 1 year postoperatively only. Additionally, there was no significant difference in postoperative Lund-Kennedy endoscopic scores among the 3 groups at all visits (Figure 1). Changes in symptoms, QoL, and endoscopic scores among the groups are shown in Figure 2.

Recurrence rate and revision surgery rate among the groups Sixteen (59.3\%), 17 (63.0\%), and 24 (88.9\%) patients suffered recurrence by 1 year postsurgery in the RESS+Draf 3, RESS, and FESS group, respectively $(P=0.03)$. At 3 years or 5 years postoperatively, the recurrence rate ranged from $95.6 \%$ to $96.1 \%$, and was not significantly different among groups (Table 2 ). Only 1 patient in each group did not eventually relapse. All patients who dropped out of the study had recurrence within 1 year postsurgery and completed the 1 year assessments. Moreover, the RESS and RESS+Draf 3 groups exhibited no difference regarding short-term (1 year) as well as long-term (5-years) recurrence rate.

No patient had revision surgery within 1-year postsurgery, but more patients in the FESS group than in the other groups had revision surgery by 3 years $(P<0.001)$ and 5 years $(P=0.02)$ postsurgery (Table 2). In 3 years, the revision surgery rates of FESS, RESS and RESS+Draf 3 groups were $38.5 \%, 0 \%$ and $8.0 \%$, respectively; whereas in 5 years, the revision surgery rates of FESS, RESS and RESS+Draf 3 groups were $45.2 \%, 17.4 \%$ and 
A

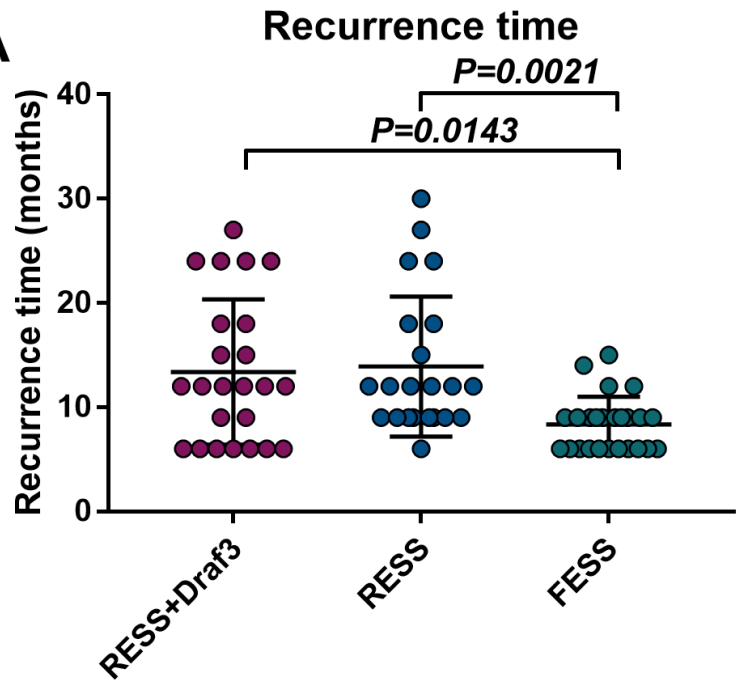

C

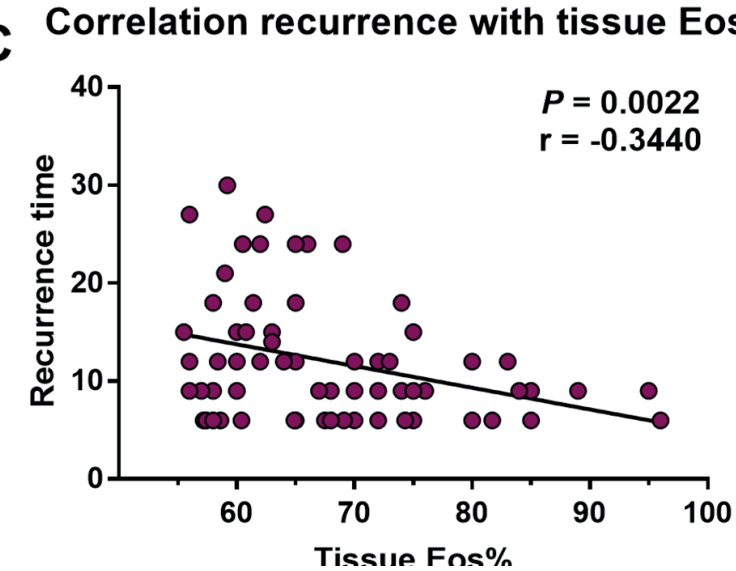

B Recurrence curve

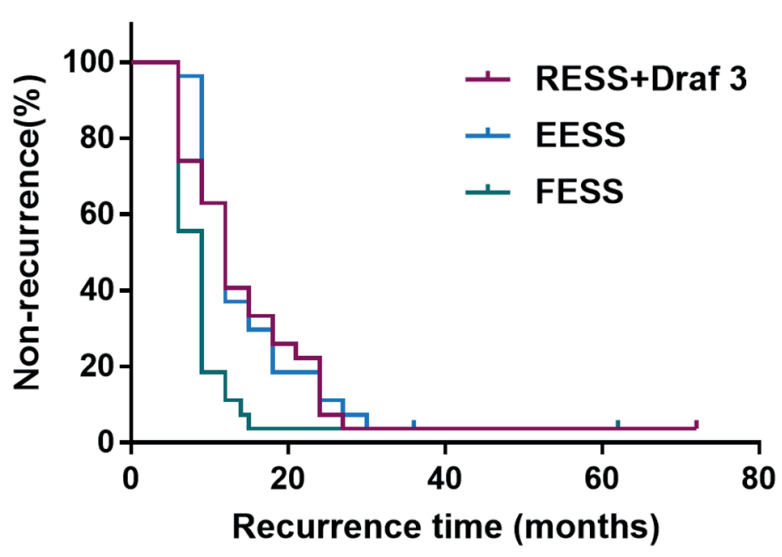

Figure 3. Comparison of recurrence time and analysis of correlations of recurrence time with tissue or blood eosinophil percentage among patients who underwent FESS, RESS, and RESS+Draf 3. FESS: functional endoscopic sinus surgery; RESS: radical endoscopic sinus surgery; Eos: eosinophils.

$16.0 \%$, respectively. Revision surgery rate did not differ between the RESS and RESS+Draf 3 groups at 1 year, 3 years, and 5 years postoperatively.

Comparison of recurrence time and related risk factors The time to recurrence after surgery was shorter in the FESS than the RESS+Draf 3 (9.0 [6.0-9.0] months versus 12.0 [6.0-19.0] months, $\mathrm{P}=0.005$ ) and RESS group (9.0 [6.0-9.0] months versus 12.0 [9.0-18.0] months, $P<0.001$ ) (Figure 3A). A Kaplan-Meier curve of the relapse-free rate in the different groups showed superior results for RESS+Draf 3 and RESS (log rank test, $\mathrm{P}=$ 0.003) (Figure 3B). Cox proportional hazards regression revealed significant differences in recurrence rate and increased tissue eosinophil percentage (Hazard rate $=1.022, \mathrm{P}=0.03$ ). There was a weak negative correlation of tissue $(r=-0.254, P=0.03)$ (Figure 3C) and peripheral blood $(r=-0.227, P=0.05)$ (Figure 3D) eosinophil percentage with recurrence time.
Clinical control of CRS and asthma at 1 year, 3 years, and 5 years postoperatively

According to clinical parameters, including symptoms and endoscopy view, patients in the RESS+Draf 3 and RESS groups had significantly better control of CRS than those in the FESS group $(P=0.009)$ at 1 year. However, no significant difference was found among the groups at 3 years and 5 years postsurgery (Figure 4A). Likewise, there was also no difference among the groups regarding clinical control of asthma at 5 -years postoperatively (Figure 4B). No significant changes were observed between baseline and 5 years in the FEV $1 \%$ results among the groups (Figure 4C). The average percentage of patients with controlled, partly controlled, and uncontrolled CRS at 5-years postoperatively was $4.0 \%, 64.9 \%$, and $31.1 \%$, respectively. The average percentage of patients with controlled, partly controlled, and uncontrolled asthma at 5-years postoperatively was $87.8 \%, 13.5 \%$, and $0 \%$ respectively, indicating markedly better control of asthma than of CRS. 
A

A
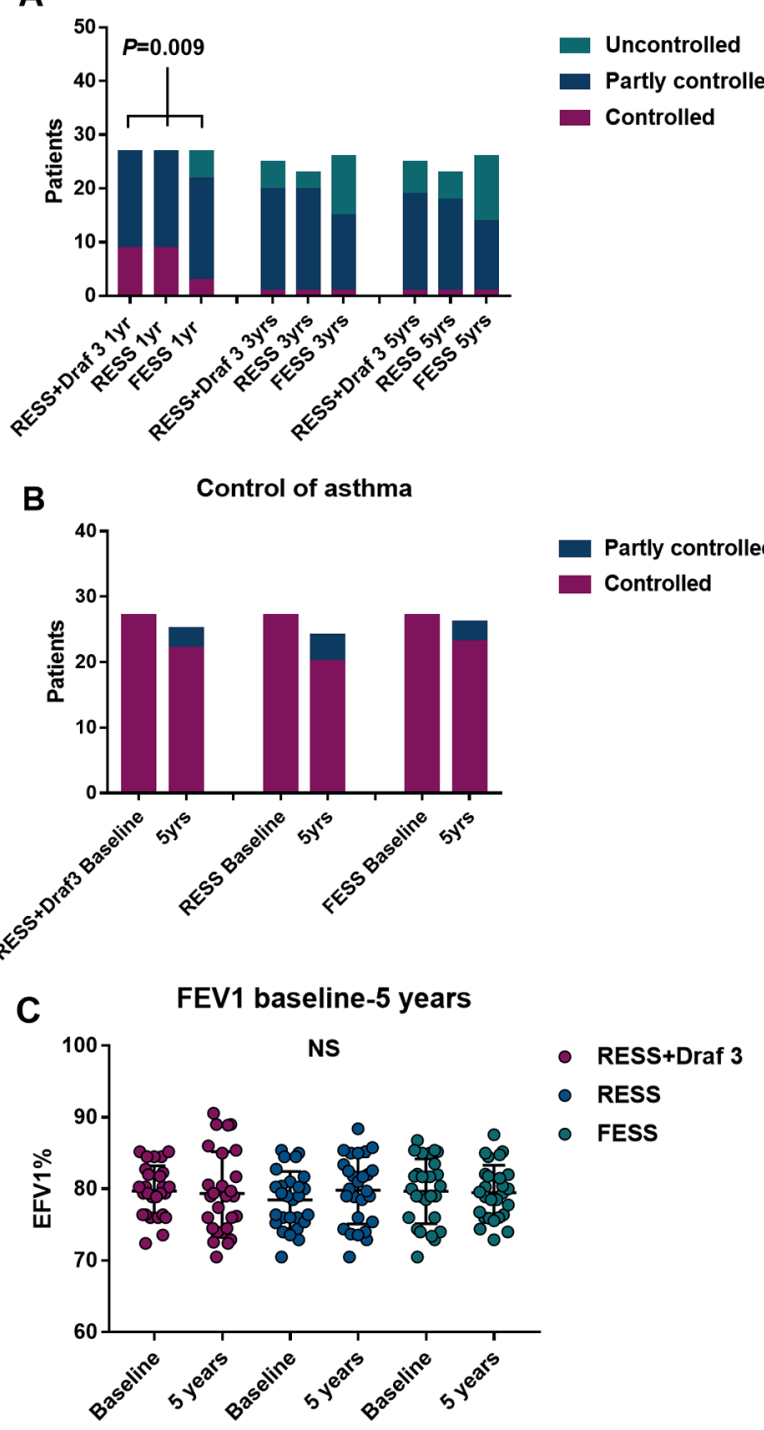

Figure 4. Assessment of clinical control of CRS and asthma in patients who underwent FESS, RESS, and RESS+Draf 3. FESS: functional endoscopic sinus surgery; RESS: radical endoscopic sinus surgery; FEV1: forced expiratory volume in 1 second.

\section{Discussion}

In this 5-year prospective study, we investigated the difference in long-term outcomes among 81 patients with refractory CRSwNP and asthma, who underwent FESS, RESS and RESS+Draf 3. RESS and RESS+Draf 3 yielded better improvement than FESS in terms of rhinorrhea, olfactory hypothyroidism, and QoL, but only in the short-term. FESS had a higher short-term recurrence rate than RESS and RESS+Draf 3, and the 3 procedures reached similar, high recurrence rates (95.65-96.1\%) by 5-years postoperatively. RESS and RESS+Draf 3 demonstrated a lower revision surgery rate than FESS in the long-term, but not at 1-year postsurgery. There was no significant difference in clinical outcome, recurrence rate, and revision surgery rate between
RESS and RESS+Draf 3 overall. However, RESS and RESS+Draf 3 had a longer recurrence time postsurgery than FESS; recurrence time negatively correlated with tissue and peripheral blood eosinophil percentage. The clinical control of CRS was markedly worse than control of asthma in the long-term.

CRS is a heterogeneous disorder with distinct pathophysiological mechanisms, this may directly influence the efficacy of medical and surgical treatments, leading to persistence or recurrence of NPs ${ }^{(13,30,31)}$. Especially, CRSwNP complicated with asthma has poorer outcomes after FESS and higher recurrence rate. The available treatment approaches, based on traditional phenotype-based classification (CRSsNP and CRSwNP) is fairly inadequate to elucidate the underlying pathophysiology in CRS and seriously hampers accurate treatment of CRS. It has been proposed that identification of CRS endotypes with appropriate biomarkers will be valuable for selecting the proper therapeutic approaches for individual patients ${ }^{(13,32)}$. To date, there are no validated biomarkers of endotypes, severity of inflammation, or therapeutic responses to medical versus surgical treatment ${ }^{(13,}$ 33). However, Lou et al. demonstrated that an absolute tissue eosinophil count $>55$ eosinophils per high power field or $>27 \%$ eosinophils per cells counted in a sinonasal tissue specimen predicted the recurrence of NPs within 2 years of sinus surgery ${ }^{(2)}$. Moreover, they showed that 5 tissue inflammatory cell patterns can contribute to objective classification of CRSwNP patients ${ }^{(3)}$. Cluster 5, comprising $37.2 \%$ of all patients, demonstrated the characteristics of eCRSwNP, with marked tissue and peripheral eosinophilia, accompanied by the highest incidence of comorbid asthma (34.6\%) and highest recurrence rate $(98.5 \%)^{(3)}$. Likewise, the presence of mucosal eosinophilia was the most important risk factor for recurrence in CRSwNP(34-37). In present study, we chose recurrent CRSwNP with asthma as a study focus, as it is the most challenging entity in clinical practice. As expected, our data showed all subjects's polyps were eosinophilic subtype ${ }^{(3)}$, the average tissue eosinophil percentage was $68.4 \pm 9.3 \%$, indicating that they were typical and strongly eosinophilic CRSwNP patients.

The type of surgery that should be used for distinct endotypes of CRSwNP is controversial(13). Several studies have confirmed that an extended or radical surgery approach may deliver better results than traditional FESS $(14,15,22,23,38-40)$. Friedman and colle-

agues first showed that extended revision decreased recurrence from $19.2 \%$ to $<5 \%$ at $18-48$ months postoperatively ${ }^{(39)}$. Likewise, in a 5-year retrospective study, Jankowski et al. reported that the recurrence rate with the radical (nasalization) procedure was $22.7 \%$, compared to $58.3 \%$ with functional ethmoidectomy ${ }^{(14)}$. Another study reported that the radical approach significantly decreased the need for revision surgery from $12.3 \%$ to $4.0 \%$ at 36 months, compared to FESS.(22) A recent study assessed the role of Draf 3 in NP recurrence and reported significantly different revision rates between FESS (37\%) and Draf 3 (7\%) over a 
12-month follow-up period ${ }^{(40)}$. Similarly, we also found a reduced revision surgery rate with the radical approach compared to traditional FESS at 3 years postsurgery. Comparatively, differences among RESS+Draf 3, RESS, and FESS were only marked at 1 year postsurgery, and by 3 and 5 years postoperatively, the recurrence rate among the groups exhibited no difference. The reason for such differences may be due to differences in study population selection and follow-up duration; standardized subject selection and long-term follow-up would help to obtain objective results. In terms of clinical outcomes, including symptoms, QoL, and endoscopic score, Chen et al.'s 1-year prospective study revealed that RESS for patients with CRSwNP and asthma may help to improve subjective olfaction and endoscopic appearance ${ }^{(15)}$. Correspondingly, RESS+Draf 3 and RESS significantly relieved patients' symptoms of rhinorrhea and loss of smell, and SNOT-22 score, as compared with FESS at 1 year postoperatively, but by 3 years postoperatively, there was no difference. LundKennedy endoscopic scores did not differ among the 3 groups in any of the follow-up surveys.

We did not detect an obvious advantage of including Draf 3 in eCRSwNP recurrence or revision surgery rate or clinical outcomes, as compared with RESS alone, and there was no difference in CRS clinical control between RESS and RESS+Draf 3, in either the short or long term. Bassiouni proposed that Draf 3 did not completely prevent polyp recurrence, but was a significant factor in reducing persistence of polyps, indicating that the procedure allowed better control of the disease ${ }^{(40)}$, based on a comparison of Draf 3 and traditional FESS. Very recently, another study reported the equivalence in outcomes between Draf IIB vs Draf 3 frontal sinusotomy for refractory chronic frontal rhinosinusitis ${ }^{(41)}$. We also concluded that the benefits of RESS+Draf 3 and RESS in reducing polyp recurrence and revision risk was similar. Thus, RESS may be more appropriate for highly eosinophilic CRSwNP disease or concomitant asthma, as including Draf 3 would prolong the operation time, exposing patients to greater risks. Of course, it is undeniable that Draf3 still has positive significance in the case of narrow ostium of frontal sinus, hyperosteogeny of frontal recess, and complex cells in frontal sinus (such as Kune type 4 frontal cell). Here we didn't observe such obvious advantage of Draf 3 currently might due to the limited sample size and the follow-up duration. Furthermore, we have to acknowledge that the medication use among groups may not reach the strict standardization which will eventually affect the clinical evaluation. Further investigation regarding the exact role of Draf 3 and other different approaches in eCRSwNP or recurrent CRSWNP combined with asthma involving more patients, longer follow-up duration and stricter standardized medication use should be addressed in the near future.

Regardless of whether a radical approach could reduce the recurrence rate or improve clinical outcomes, widening of the sinuses and olfactory area might guarantee a longer recurrence- free time. Moreover, tissue and peripheral blood eosinophil percentage correlated negatively with recurrence time, in agreement with Tosun et al's reports that polyp recurrence correlated with the eosinophilic content of polyps ${ }^{(42)}$. Although eCRSwNP is the most aggressive CRS subtype, glucocorticosteroids clearly work better in an eosinophilic environment and many studies have reported that an eosinophilic characteristic was associated with good responsiveness to glucocorticosteroid therapy ${ }^{(6,7,43)}$, suggesting that persistent usage of systemic or intranasal steroid therapy is probably a good strategy for management of the condition. However, eosinophilic inflammation can be so strong that steroids are no longer effective ${ }^{(44,45)}$, and typically, patients with the highest degree of inflammation use the most glucocorticosteroids, leading to an insufficient outcome and recurrence of NPs ${ }^{(44,45)}$. Indeed, in the present study, $96.3 \%$ patients overall had recurrence within 3 years postoperatively, although two-thirds had undergone extended surgeries, suggesting the potential insufficiency or low efficacy of glucocorticosteroid therapy employed under these conditions. It is possible that despite continuous postoperative use of intranasal budesonide for at least 6 months until the patients had achieved good control, or additional use of oral methylprednisolone in patients not responding well to intranasal budesonide, this treatment was not very effective for several reasons. Firstly, all the subjects enrolled in the present study suffered from eCRSwNP, which is the most aggressive CRS subtype and particularly liable to relapse. Secondly, although steroid spray was used, it may not have penetrated into the sinuses, leading to under treatment and possibly masking any optimal benefits of the radical approach especially Draf 3. On this issue, recent evidence indicates that corticosteroid delivered by nasal irrigation is superior to simple intranasal spray postsurgery in CRS patients ${ }^{(46)}$. The present study was designed with the EPOS 2007/2012 guidelines $^{(1,19)}$ and conducted from January1, 2010 to October 31, 2013 when the treatment strategy of adding the steroid to the irrigation was not explicitly recommended by the EPOS guidelines. Thus, in the present study all the enrolled patients were treated postoperatively with budesonide spray and nasal irrigation administered separately, rather than with the steroid added to the irrigation. Further comparative studies where patients are treated with adequate post-operative steroid irrigations will be addressed to uncover the true effect of different surgical approaches in recurrent CRSwNP.

Furthermore, clinical control of CRS was far less efficient than that of asthma in the present study, possibly reflecting the much less attention that patients as well as doctors pay to CRS than to asthma. In this regard, there is an urgent need for both the patients and the doctors to be educated on the importance of systematic treatment of CRS.

The unaffected mucosa-sparing approach was adopted for all surgical procedures. We cannot exclude that such approaches 
might increase the risk of polyp recurrence after surgery, as a recent study proved that complete removal of diseased mucosa from the paranasal sinuses (reboot approach) significantly reduced the recurrence of NPs for 30 months postoperatively, compared to the current mucosa-sparing approach, in type 2 inflammatory CRSwNP(47). Further evaluation of the immunological endotypes and the corresponding surgery outcomes should be addressed in future.

Removal of the MT guarantees adequate space for ventilation and good drainage for sinuses. Middle turbinectomy may also increase airflow to the olfactory clefts and allow more odorant particles to stimulate the olfactory nerve fiber. Moreover, in terms of the "inflammatory load hypothesis"(48), radical surgery could reduce the inflammatory load in the paranasal sinuses and provide a wide and patent sinus ostia, thereby enhancing symptomatic relief and facilitating better topical management. A previous study reported that the volume of the space between the midportion of the septum and the MT correlated strongly with olfaction ${ }^{(49)}$. Chen et al. ${ }^{(15)}$ further demonstrated that extensive surgery involving MT removal in patients with CRSwNP and with asthma may help to improve their subjective olfaction as well as the endoscopic appearance 1 year postsurgery. We found that patients in both the RESS+Draf 3 group and RESS group had significantly decreased olfactory scores than those in the FESS group only at 1 year, but not at 3 or 5 years postoperatively, indicating that the olfaction improvement derived from radical surgery was short-term.

\section{Conclusion}

Patients with CRSwNP and asthma have poorer outcomes after FESS and higher recurrence rate. To date, no recognized surgical strategy is available for CRSwNP with asthma. Here we investigated the long-term clinical outcomes of extended surgical strategies for patients with recurrent CRSwNP and asthma. We concluded that on the premise of continuous medication, radical surgery could prolong time to recurrence, improve olfaction, rhinorrhea, and QoL in the short-term. The combination with Draf 3 did not improve the outcomes obtained with RESS alone. Although RESS was shown to be the best option for patients with CRSwNP and asthma, these findings need to be confirmed in further studies involving more patients, longer follow-up duration and stricter standardized medication use especially the adequate steroid irrigations.

\section{Acknowledgements}

This work was supported by grants from the program for National Key R\&D Program of China (2016YFC0905200, 2018YFC0116801), the National Natural Science Foundation of China $(81570895,81420108009,81400444,81870698$, and 81630023), Changjiang Scholars and Innovative Research Team (IRT13082), the Special Fund of Capital Health Development (2011-1017-06,2011-1017-02), the Special Fund of Sanitation Elite Reconstruction of Beijing (2009-2-007), Beijing Municipal Administration of Hospitals' Mission Plan (SML20150203), Capital Citizenry Health Program (z161100000116062), and Beijing Municipal Administration of Hospitals'Yangfan Plan (XMLX201816).

\section{Authorship contribution}

LZ and CW were responsible for the overall design and subject recruitment. CW performed all endoscopic sinus surgeries and postoperative follow-up. YZ and LZ assessed all clinical outcomes. YZ, YG, KW, HL, and YM contributed to acquiring, analyzing, and interpreting the data and preparing the manuscript.

\section{Conflict of interest}

The authors state that they have no conflict of interest.

\section{References}

1. Fokkens WJ, Lund VJ, Mullol J, et al. European Position Paper on Rhinosinusitis and Nasal Polyps 2012. Rhinol Suppl. 2012, 23:3 p preceding table of contents, 1-298.

2. Lou H, Meng Y, Piao Y, Wang C, Zhang L, Bachert $C$. Predictive significance of tissue eosinophilia for nasal polyp recurrence in the Chinese population. Am J Rhinol Allergy. 2015, 29:350-356.

3. Lou H, Meng Y, Piao Y, et al. Cellular phenotyping of chronic rhinosinusitis with nasal polyps. Rhinology. 2016, 54:150-159.

4. Newman LJ, Platts-Mills TA, Phillips CD, Hazen KC, Gross CW. Chronic sinusitis. Relationship of computed tomographic findings to allergy, asthma, and eosinophilia. JAMA. 1994, 271:363-367.

5. Hellquist HB. Nasal polyps update Histopathology. Allergy and asthma pro- ceedings. 1996, 17:237-242.

6. Kirtsreesakul V, Atchariyasathian V. Nasal polyposis: role of allergy on therapeutic response of eosinophil- and noneosinophil-dominated inflammation. Am J Rhinol. 2006, 20:95-100.

7. Wang $C$, Lou $H$, Wang $X$, et al. Effect of budesonide transnasal nebulization in patients with eosinophilic chronic rhinosinusitis with nasal polyps. J Allergy Clin Immunol. 2015, 135:922-929 e926.

8. Hastan D, Fokkens WJ, Bachert C, et al. Chronic rhinosinusitis in Europe--an underestimated disease. A GALEN study. Allergy. 2011, 66:1216-1223.

9. Stammberger H, Posawetz W. Functional endoscopic sinus surgery. Concept, indications and results of the Messerklinger technique. Eur Arch Otorhinolaryngol. 1990, 247:63-76.
10. Hosemann W, Gode U, Wigand ME. Indications, technique and results of endonasal endoscopic ethmoidectomy. Acta Otorhinolaryngol Belg. 1993, 47:73-83.

11. Govindaraj S, Adappa ND, Kennedy DW. Endoscopic sinus surgery: evolution and technical innovations. J Laryngol Otol. 2010, 124:242-250.

12. Kennedy DW. Technical innovations and the evolution of endoscopic sinus surgery. Ann Otol Rhinol Laryngol Suppl. 2006, 196:3-12.

13. Bachert C, Akdis CA. Phenotypes and Emerging Endotypes of Chronic Rhinosinusitis. J Allergy Clin Immunol Pract. 2016, 4:621-628.

14. Jankowski R, Pigret D, Decroocq F, Blum A, Gillet P. Comparison of radical (nasalisation) and functional ethmoidectomy in patients with severe sinonasal polyposis. A retrospective study. Rev Laryngol Otol Rhinol 
(Bord). 2006, 127:131-140

15. Chen FH, Deng J, Hong HY, et al. Extensive versus functional endoscopic sinus surgery for chronic rhinosinusitis with nasal polyps and asthma: A 1-year study. Am J Rhinol Allergy. 2016, 30:143-148.

16. Morrissey DK, Bassiouni A, Psaltis AJ, Naidoo Y, Wormald PJ. Outcomes of modified endoscopic Lothrop in aspirin-exacerbated respiratory disease with nasal polyposis. Int Forum Allergy Rhinol. 2016, 6:820-825.

17. Wormald PJ, Bassiouni A, Callejas CA, et al. The International Classification of the radiological Complexity (ICC) of frontal recess and frontal sinus. Int Forum Allergy Rhinol. 2017;7:332-337.

18. Zhang X, Ye T, Huang Q, et al. Clinical predictors of frontal ostium restenosis after Draf3 procedure for refractory chronic rhinosinusitis. Am J Rhinol Allergy. 2018 Jul; 32(4):287-293.

19. Fokkens W, Lund V, Mullol J. European position paper on rhinosinusitis and nasal polyps 2007. Rhinol Suppl. 2007, 20:1-136.

20. Bateman ED, Hurd SS, Barnes PJ ,et al. Global strategy for asthma management and prevention: GINA executive summary. Eur Respir J. 2008, 31:143-178.

21. Seiberling K, Floreani S, Robinson S, Wormald PJ. Endoscopic management of frontal sinus osteomas revisited. Am J Rhinol Allergy. 2009, 23:331-336.

22. Masterson L, Tanweer F, Bueser $T$, Leong P. Extensive endoscopic sinus surgery: does this reduce the revision rate for nasal polyposis? Eur Arch Otorhinolaryngol. 2010, 267:1557-1561

23. Shen PH, Weitzel EK, Lai JT, Wormald PJ, Lin $\mathrm{CH}$. Retrospective study of full-house functional endoscopic sinus surgery for revision endoscopic sinus surgery. Int Forum Allergy Rhinol. 2011, 1:498-503.

24. Wormald PJ. Salvage frontal sinus surgery: the endoscopic modified Lothrop procedure. Laryngoscope. 2003, 113:276-283.

25. DeConde AS, Bodner TE, Mace JC, Smith TL. Response shift in quality of life after endoscopic sinus surgery for chronic rhinosinusitis. JAMA Otolaryngol Head Neck Surg. 2014, 140:712-719.

26. Stjarne $\mathrm{P}$, Olsson $\mathrm{P}$, Alenius M. Use of mometasone furoate to prevent polyp relapse after endoscopic sinus surgery. Arch Otolaryngol Head Neck Surg. 2009, 135:296302.

27. Lund VJ, Kennedy DW. Quantification for staging sinusitis. The Staging and Therapy Group. Ann Otol Rhinol Laryngol Suppl. 1995, 167:17-21.
28. Lund VJ, Kennedy DW. Staging for rhinosinusitis. Otolaryngol Head Neck Surg. 1997 117(3 Pt 2):S35-40.

29. Nathan RA, Sorkness CA, Kosinski M, et al. Development of the asthma control test: a survey for assessing asthma control. J Allergy Clin Immunol. 2004, 113:59-65.

30. Akdis CA, Bachert C, Cingi C, et al. Endotypes and phenotypes of chronic rhinosinusitis: a PRACTALL document of the European Academy of Allergy and Clinical Immunology and the American Academy of Allergy, Asthma \& Immunology. J Allergy Clin Immunol. 2013, 131:1479-1490.

31. Cao PP, Wang ZC, Schleimer RP, Liu Z Pathophysiologic mechanisms of chronic rhinosinusitis and their roles in emerging disease endotypes. Ann Allergy Asthma Immunol. 2019, 122:33-40.

32. Bachert C, Zhang N, Hellings PW, Bousquet J. Endotype-driven care pathways in patients with chronic rhinosinusitis. Allergy Clin Immunol. 2018, 141:1543-1551.

33. Stevens WW, Schleimer RP, Kern RC. Chronic Rhinosinusitis with Nasal Polyps. J Allergy Clin Immunol Pract. 2016, 4:565-572.

34. Ikeda K, Shiozawa A, Ono N, et al. Subclassification of chronic rhinosinusitis with nasal polyp based on eosinophil and neutrophil. Laryngoscope. 2013, 123:E1-9.

35. Nakayama T, Yoshikawa M, Asaka D, et al Mucosal eosinophilia and recurrence of nasal polyps - new classification of chronic rhinosinusitis. Rhinology. 2011, 49:392-396.

36. Matsuwaki Y, Ookushi T, Asaka D, et al. Chronic rhinosinusitis: risk factors for the recurrence of chronic rhinosinusitis based on 5-year follow-up after endoscopic sinus surgery. Int Arch Allergy Immunol. 2008, 146 Suppl 1:77-81.

37. Stoop AE, van der Heijden HA, Biewenga J, van der Baan S. Eosinophils in nasal polyps and nasal mucosa: an immunohistochemical study. J Allergy Clin Immunol. 1993, 91:616-622

38. Videler WJ, van Drunen CM, van der Meulen FW, Fokkens WJ. Radical surgery: effect on quality of life and pain in chronic rhinosinusitis. Otolaryngol Head Neck Surg. 2007 136:261-267.

39. Friedman WH, Katsantonis GP. Transantral revision of recurrent maxillary and ethmoidal disease following functional intranasal surgery. Otolaryngol Head Neck Surg. 1992 106:367-371.

40. Bassiouni A, Wormald PJ. Role of fronta sinus surgery in nasal polyp recurrence. Laryngoscope. 2013, 123:36-41.

41. Patel VS, Choby G, Shih LC, Patel ZM, Nayak
$\mathrm{JV}$, Hwang PH. Equivalence in outcomes between Draf 2B vs Draf 3 frontal sinusotomy for refractory chronic frontal rhinosinusitis. Int Forum Allergy Rhinol. 2018, 8:25-31.

42. Tosun F, Arslan HH, Karslioglu Y, Deveci MS, Durmaz A. Relationship between postoperative recurrence rate and eosinophil density of nasal polyps. Ann Otol Rhinol Laryngol. 2010, 119:455-459.

43. Burgel PR, Cardell LO, Ueki IF, Nadel JA Intranasal steroids decrease eosinophils but not mucin expression in nasal polyps. Eur Respir J. 2004, 24:594-600.

44. Jun YJ, Park SJ, Kim TH, Lee SH, Lee KJ, Hwang SM. Expression of 11 beta-hydroxysteroid dehydrogenase 1 and 2 in patients with chronic rhinosinusitis and their possible contribution to local glucocorticoid activation in sinus mucosa. J Allergy Clin Immunol. 2014, 134:926-934 e926.

45. Milara J, Peiro T, Armengot M, et al. Mucin 1 downregulation associates with corticosteroid resistance in chronic rhinosinusitis with nasal polyps. J Allergy Clin Immunol. 2015, 135:470-476.

46. Harvey RJ, Snidvongs K, Kalish LH, Oakley GM, Sacks R. Corticosteroid nasal irrigations are more effective than simple sprays in a randomized double-blinded placebocontrolled trial for chronic rhinosinusitis after sinus surgery. Int Forum Allergy Rhinol. 2018, 8:461-470

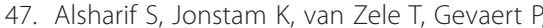
Holtappels G, Bachert C. Endoscopic Sinus Surgery for Type-2 CRS wNP: An EndotypeBased Retrospective Study. Laryngoscope. 2019;129(6):1286-1292.

48. Bassiouni A, Naidoo Y, Wormald PJ. When FESS fails: the inflammatory load hypothesis in refractory chronic rhinosinusitis. Laryngoscope. 2012, 122:460-466.

49. Leopold DA. The relationship between nasal anatomy and human olfaction. Laryngoscope. 1988, 98:1232-1238.

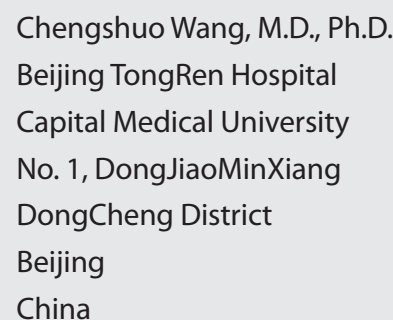

E-mail:wangcs830@126.com 